\section{Thermal conduction through a molecule}

To cite this article: A. Buldum et al 1999 EPL 47208

View the article online for updates and enhancements.
Related content

- Quantum heat transfer through an atomic
$\frac{\text { wire }}{\text { A Buldum, S Ciraci and C Y Fong }}$
- Quantum effects in electrical and thermal
$\frac{\text { transport throughnanowires }}{\text { S Ciraci, A Buldum and Inder P Batra }}$
- Statistics of conductance oscillations of a
$\frac{\text { quantum dot in the Coulomb-blockade }}{\text { regime }}$
F. Simmel, T. Heinzel and D. A. Wharam

\section{Recent citations}

- Vibrational Heat Transport in Molecular
$\frac{\text { Junctions }}{\text { Dvira Segal and Bijay Kumar Agarwalla }}$
- Quantum bottlenecks and unidirectional
$\frac{\text { energy flow in molecules }}{\text { David M. Leitner and Hari Datt Pandey }}$
- Quantum heat transport of a two-qubit
svstem: Interplay between system-bath
coherence and qubit-qubit coherence
Akihito Kato and Yoshitaka Tanimura


Europhys. Lett., 47 (2), pp. 208-212 (1999)

\title{
Thermal conduction through a molecule
}

\author{
A. Buldum, D. M. Leitner(*) and S. Ciraci \\ Department of Physics, Bilkent University - Bilkent 06533, Ankara, Turkey
}

(received 11 December 1998; accepted in final form 3 May 1999)

PACS. 63.22 $+\mathrm{m}-$ Phonons in low-dimensional structures and small particles.

PACS. 44.10+i - Heat conduction.

PACS. $61.46+\mathrm{w}-$ Clusters, nanoparticles, and nanocrystalline materials.

\begin{abstract}
The quantum features of phononic thermal conduction through a molecule between two reservoirs have been studied in the weak-coupling limit. As opposed to ballistic heat transfer through a uniform bridge or atomic chain strongly coupled to reservoirs investigated earlier, thermal conductance in the present case shows a nonlinear temperature dependence that is sensitive to the mode frequencies of the molecule.
\end{abstract}

Recent theoretical studies have shown that at low temperature the phononic thermal conductance of a uniform dielectric wire or an atomic chain is universal, material independent, and directly proportional to the temperature [1-3]. This universal value has been denoted as the quantum of thermal conductance. A molecule (or a nanoparticle or atomic chain) between two reservoirs is also an important system from the mesoscopic heat conduction point of view. Owing to the finite level spacings, $\Delta \omega_{q}=\omega_{q+1}-\omega_{q}$, of its mode frequencies and its relatively small size, being smaller than the mean distance between two consecutive inelastic scatterings of phonons, the quantization and coherence of the vibrational states become pronounced. Consequently, thermal conduction through a molecule is expected to exhibit novel quantum features. The heat transfer described in the above physical system, i.e., a molecule between two reservoirs, is relevant for tribology [4]. Heat generated in friction is dissipated into the moving objects (or reservoirs) through lubricant molecules. The lubricant molecules are typically weakly coupled to the reservoirs. The understanding of various events involved in the energy dissipation process and developing a theory which allows one to carry out a quantitative analysis of atomic (or molecular) scale lubrication are important for tribology. The present work deals with phononic thermal conduction through a molecule that is weakly coupled to two reservoirs and aims to reveal quantum features of the thermal conductance.

Our calculation of the quantum-mechanical thermal conductance due to phonons for the system illustrated in fig. 1 begins with the evaluation of the rate of energy transfer from the

$\left({ }^{*}\right)$ Permanent address: UCSD Department of Chemistry and Biochemistry, La Jolla, CA 920930339, USA.

(C) EDP Sciences 


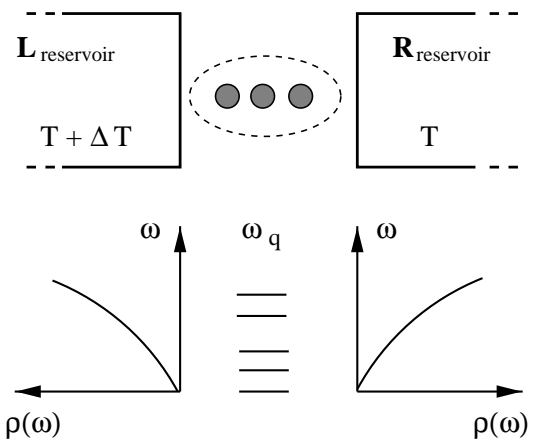

Fig. 1. - A schematic illustration of the reservoirs and a molecule through which heat is transferred. The densities of vibrational frequencies of the left $(\mathrm{L})$ and right $(\mathrm{R})$ reservoirs, $\rho(\omega)$, and discrete frequencies of the molecule, $\omega_{q}$, are also shown.

higher-temperature to the lower-temperature reservoir via the molecule,

$$
H^{+}=\sum_{l} \hbar \omega_{l} \Gamma_{l}
$$

where $\omega_{l}$ is the frequency of mode $l$ of the higher-temperature reservoir, e.g., the left one depicted in fig. 1. We can similarly write down the rate of energy transfer in the other direction, $H^{-}$. We address the limit where the molecule is weakly coupled to the reservoirs; for example, the molecule might be physisorbed to the reservoirs, or the distance between it and the reservoirs might be sufficiently large so that the interaction is not too strong. When the molecule is weakly coupled to the reservoirs, the decay rate, $\Gamma_{l}$, of mode $l$ is given to lowest order perturbation theory by

$$
\Gamma_{l}=\frac{2 \pi}{\hbar^{2}}|\mathcal{V}|^{2} \rho\left(\omega_{l}\right)
$$

where $\mathcal{V}$, defined below, couples the left to right reservoirs via coupling to the molecule. For simplicity we assume that other than their temperatures the left and right reservoirs are identical, so that the mode densities, $\rho(\omega)$, of both reservoirs at the same mode frequency are the same. We express the operator, $\widehat{V}$, coupling each reservoir to the molecule to lowest order as

$$
\widehat{V}=\sum_{l, q} \mathcal{T}_{l q}\left(\hat{a}_{l}^{\dagger} \hat{a}_{q}+\hat{a}_{l} \hat{a}_{q}^{\dagger}\right)+\sum_{r, q} \mathcal{T}_{r q}\left(\hat{a}_{r}^{\dagger} \hat{a}_{q}+\hat{a}_{r} \hat{a}_{q}^{\dagger}\right),
$$

where $l$ and $r$ are modes of the left and right reservoirs, respectively, and $q$ is a mode of the molecule. In the present study we consider only linear coupling between modes of the reservoirs and the molecule, neglecting the smaller nonlinear terms. We also assume that at the low temperatures we are interested in we can neglect anharmonic coupling among the modes within the molecule. This is, in fact, a safe assumption for even moderate-sized molecules with, say, tens of atoms [5]. We then have for the coupling

$$
|\mathcal{V}|^{2}=\hbar^{-2} \sum_{r}\left|\sum_{q} \frac{\left(\bar{n}_{r}+1\right)^{1 / 2}\left(\bar{n}_{q}+1\right) \bar{n}_{l}^{1 / 2} \mathcal{T}_{l q} \mathcal{T}_{q r}}{\omega-\omega_{q}+i \gamma_{q}}\right|^{2}
$$

where we introduce the most probable occupation numbers, $\bar{n}_{l}, \bar{n}_{r}$, and $\bar{n}_{q}$, for modes of the left and right reservoirs, and the molecule, respectively, which we calculate from the Planck 
distribution. Equation (4) describes a Raman process, where phonons are transferred from one reservoir to the other by scattering through the intermediate states of the molecule. The linear coupling coefficients, $\mathcal{T}$, scale as the square root of the product of the frequencies of the coupled modes [6],

$$
\mathcal{T}_{l q}=a \hbar\left(\omega_{l} \omega_{q}\right)^{1 / 2}
$$

The dimensionless coefficient $a$ describes the coupling of the molecule to the reservoir, and will depend on the kind of bonding and the nature and size of the molecule and reservoir [7]. We will assume that $a$ has been calculated for a particular system. The imaginary part, $\gamma_{q}$, in eq. (4) corresponds to the decay rate from mode $q$ due to coupling of the molecular modes to the reservoirs. It is given by the golden rule in this weak-coupling limit, $\gamma_{q}=$ $\frac{2 \pi}{\hbar^{2}} \sum_{l}\left|\mathcal{V}_{l q}\right|^{2} \rho\left(\omega_{q}\right)$, where $\mathcal{V}_{l q}$ are matrix elements coupling states of the reservoirs to mode $q$. We assume throughout that the energy levels of the molecule are narrow and resolvable, i.e., $\gamma_{q}+\gamma_{q^{\prime}} \ll \omega_{q}-\omega_{q^{\prime}}, q \neq q^{\prime}$. Since the mode density of the reservoirs is taken to be large, we replace the sum in eq. (1) with an integral over the mode frequencies of the left, higher-temperature reservoir,

$$
H^{+}=\frac{2 \pi}{\hbar^{2}} \int \mathrm{d} \omega \hbar \omega \rho^{2}(\omega)|\mathcal{V}|^{2}
$$

We then find upon integration

$$
H^{+}=\frac{\pi}{\hbar^{4}} a^{2} b \sum_{q} \bar{n}_{l}\left(\omega_{q}\right)\left(\bar{n}_{q}\left(\omega_{q}\right)+1\right)\left(\hbar \omega_{q}\right)^{5},
$$

where the sum is taken over molecular modes. In eq. (7), we use the Debye approximation for the mode density of the reservoir, $\rho(\omega)=b \omega^{2}$, where $b=V / 2 \pi^{2} v_{\mathrm{L}, \mathrm{T}}^{3}$ for $\omega \leq \omega_{\mathrm{D}} ; V$ is the volume of the reservoir and $v_{\mathrm{L}, \mathrm{T}}$ is the longitudinal or transverse sound velocity through it. We can also generalize to treat coupling to surface modes, whereby $\rho_{\mathrm{s}}(\omega)=b_{\mathrm{s}} \omega$. We take $a_{\mathrm{s}}$ to describe coupling to surface modes in eq. (5).

Having calculated the energy transfer rate, we obtain the thermal conductance as $\kappa=$ $\mathrm{d} H^{+} / \mathrm{d} T$, where $T$ is the temperature [2]; this is just the limit of taking $\Delta T$ between the reservoirs to be very small. Writing $x_{q}=\hbar \omega_{q} / k_{\mathrm{B}} T$, the thermal conductance, $\kappa$, reads

$$
\kappa=\frac{\pi a^{2} b}{\hbar^{4}} k_{\mathrm{B}}^{5} T^{4} \sum_{q} \frac{x_{q}^{6} \mathrm{e}^{x_{q}}\left(\mathrm{e}^{x_{q}}+1\right)}{\left(\mathrm{e}^{x_{q}}-1\right)^{3}}+\frac{\pi a_{\mathrm{s}}^{2} b_{\mathrm{s}}}{\hbar^{3}} k_{\mathrm{B}}^{4} T^{3} \sum_{q} \frac{x_{q}^{5} \mathrm{e}^{x_{q}}\left(\mathrm{e}^{x_{q}}+1\right)}{\left(\mathrm{e}^{x_{q}}-1\right)^{3}}
$$

where $k_{\mathrm{B}}$ is the Boltzmann constant. The first sum accounts for coupling to the bulk modes of the reservoirs and the latter for coupling to their surface modes.

We note two characteristics of the thermal conductance for a molecule weakly coupled to two reservoirs, as described by eq. (8). First, the dependence of thermal conductance on temperature is not simply linear, as is the case of a molecule strongly coupled to each reservoir [1-3]. Second, the low-temperature thermal conductance is strongly influenced by the individual mode frequencies of the molecule, particularly the lowest-frequency modes. Both of these features will be apparent in the following specific numerical example.

We now apply the above formalism to calculate the thermal conductance for a particular model system consisting of a chain of 3 atoms between two reservoirs. Each reservoir is represented by 7 atomic planes. The whole system contains 451 atoms. The interatomic distances within the chain, between the chain and slab atoms and within the slab are taken to be 3.50, 2.65 and $2.56 \mathrm{~A}$, respectively. Interatomic interactions are derived from an empirical potential [8], which we take here to be a prototype model potential for an electrical insulator, 

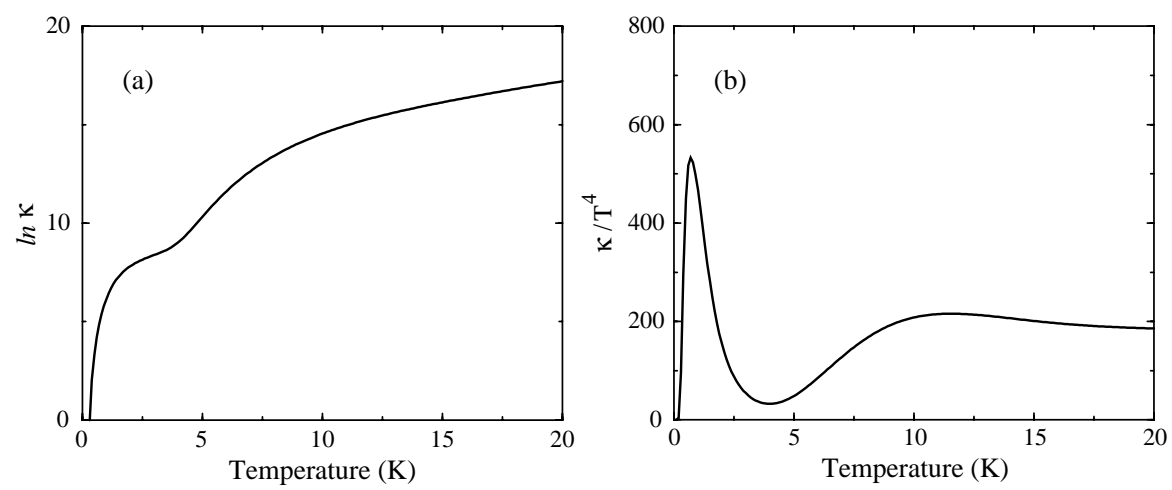

Fig. 2. - (a) Thermal conductance, $\kappa$, in units of $\pi a^{2} b k_{\mathrm{B}}^{5} / \hbar^{4}$ and (b) in units of $\pi a^{2} b k_{\mathrm{B}}^{5} / \hbar^{4} T^{4}$. The effect of discrete levels of conductor, and particularly the gap between the low-frequency transverse modes and higher-frequency longitudinal modes, is seen to be sizable at low temperature.

and the dynamical matrix of the system is diagonalized to obtain the modes. Because of the reduced number of nearest neighbors, coupling between the chain and the reservoirs is not as strong as coupling within the bulk. We have also stretched the bonds between the molecule and reservoirs to weaken the interaction between them. While this model is rather simplistic, we can nevertheless use the eigenmodes computed for this system to illustrate the effects of the discrete and sparse spectrum of a molecule on its thermal conductance due to lattice vibrations.

The lowest modes for this system are found to have contributions from either the atoms of the reservoirs or the atoms of the molecule, but very little of both. Expressing the lowest three "molecular" modes in terms of the amplitudes of all the atoms of the system, we find that over $95 \%$ of the amplitude is accounted for by the atoms of the molecule. These lowest modes are transverse, accounting for the lowest 3 (doubly degenerate) frequencies at 3.3, 3.6 and $6.7 \mathrm{~K}$. These modes are thus quite well localized to the molecule, which is apparently only weakly coupled to the reservoirs. From the widths of the narrow peaks arising from the small admixture of amplitude of the reservoir atoms we estimate $a \approx 0.02$. The three longitudinal modes, all above $50 \mathrm{~K}$, are much more broadened due to apparently stronger coupling to the reservoirs. For this system, the weak-coupling limit is thus appropriate for describing thermal conductance due to phonons at low temperatures.

In fig. 2 we plot the results of the thermal conductance as a function of temperature using eq. (8). We keep only the first sum in eq. (8) for simplicity, thereby assuming that coupling to bulk modes dominates the thermal conductance. Since we are interested in scaling with temperature and effects of a small number of conducting states and not on very system-specific parameters, we express the conductance in units of $a^{2} b$. From fig. 2(a) we observe that the thermal conductance rises rapidly until about $2 \mathrm{~K}$, then more slowly until about $4 \mathrm{~K}$, then rises rapidly again, as the effects of the much higher-frequency longitudinal modes begin to influence $\kappa$. The effect of the sum over the mode frequencies of the molecule can be seen by dividing $\kappa$ by $T^{4}$. We then see in fig. 2(b) the dramatic effect of the large gap in energy between the low-energy heat conducting states due to the transverse modes, and the high-energy states due to the longitudinal modes.

In conclusion, we have presented a theory for low-temperature thermal conductance due to phonon transport through a molecule. We describe this as a Raman process, whereby phonons are transferred from one reservoir to the other via transitions to resonant and off- 
resonant states of the molecule that connects them. This is also analagous to the "vibrational superexchange" mechanism for energy flow in large molecules [9-11]. Such a model is applicable to studies of nanotribology where the molecule is a lubricant, though we have here considered only an individual lubricant and not an ensemble. For the latter, potentially interesting effects due to intermolecular interactions or quantum coherences could arise. For our system of a single molecule bridging two reservoirs, eq. (8) expresses the thermal conductance in terms of the temperature, mode frequencies of the molecule, and the interaction strength. As opposed to the case of conductance due to ballistic phonons through bridges strongly coupled to reservoirs investigated earlier [1-3], thermal conductance through a molecule varies nonlinearly with temperature. We have calculated the thermal conductance for a particular model system, a 3-atom chain coupled on each end to a reservoir. There is a sizable gap in the frequency spectrum of the 3-atom molecule, which gives rise to the interesting variation of the thermal conductance with temperature observed in fig. 2.

DML acknowledges fruitful discussions with P. G. WOLYNES.

\section{REFERENCES}

[1] Rego L. G. C. and Kirczenow G., Phys. Rev. Lett., 81 (1998) 232.

[2] Angelescu D. E., Cross M. C. and Roukes M. L., Superlatt. Microstruct., 23 (1998) 673.

[3] Buldum A., Ciraci S. and Fong C. Y., submitted to Phys. Rev. B.

[4] Buldum A., Leitner D. M. and Ciraci S., to be published in Phys. Rev. B, 59 (1999).

[5] Logan D. E. and Wolynes P. G., J. Chem. Phys., 93 (1990) 4994; Leitner D. M. and Wolynes P. G., J. Chem. Phys., 101 (1996) 541; Chem. Phys. Lett., 280 (1997) 411.

[6] Madsen D., Pearman R. and Gruebele M., J. Chem. Phys., 106 (1997) 5874.

[7] Leitner D. M. and Wolynes P. G., to be published.

[8] Erkoc S., Z. Phys. D, 32 (1994) 257; Mehrez H., Ciraci S., Fong C. Y. and Erkoc S., J. Phys. Condens. Matter, 9 (1997) 10843.

[9] Leitner D. M. and Wolynes P. G., Phys. Rev. Lett., 76 (1996) 216.

[10] Stuchebrukhov A. A. and Marcus R. A., J. Chem. Phys., 98 (1993) 6044.

[11] Heller E. J., J. Phys. Chem., 99 (1995) 2625. 